\title{
Genetic analyses place most Spanish isolates of Beauveria bassiana in a molecular group with word-wide distribution
}

\author{
Inmaculada Garrido-Jurado 1,3, Marcela Márquez², , Almudena Ortiz-Urquiza', Cándido Santiago-Álvarez', \\ Enrique A Iturriaga ${ }^{2}$, Enrique Quesada-Moraga ${ }^{1}$, Enrique Monte ${ }^{3 *}$ and Rosa Hermosa ${ }^{3}$
}

\begin{abstract}
Background: The entomopathogenic anamorphic fungus Beauveria bassiana is currently used as a biocontrol agent (BCA) of insects. Fifty-seven Beauveria bassiana isolates -53 from Spain- were characterized, integrating group I intron insertion patterns at the $3^{\prime}$-end of the nuclear large subunit ribosomal gene (LSU rDNA) and elongation factor 1-alpha (EF1- $\alpha$ ) phylogenetic information, in order to assess the genetic structure and diversity of this Spanish collection of B. bassiana.

Results: Group I intron genotype analysis was based on the four highly conserved insertion sites of the LSU (Ec2653, Ec2449, Ec2066, Ec1921). Of the 16 possible combinations/genotypes, only four were detected, two of which were predominant, containing 44 and 9 members out of 57 isolates, respectively. Interestingly, the members of the latter two genotypes showed unique differences in their growth temperatures. In follow, EF1- $\alpha$ phylogeny served to classify most of the strains in the B. bassiana s.s. (sensu stricto) group and separate them into 5 molecular subgroups, all of which contained a group I intron belonging to the IC1 subtype at the EC1921 position. A number of parameters such as thermal growth or origin (host, geographic location and climatic conditions) were also examined but in general no association could be found.
\end{abstract}

Conclusion: Most Spanish B. bassiana isolates (77.2\%) are grouped into a major phylogenetic subgroup with wordwide distribution. However, high phylogenetic diversity was also detected among Spanish isolates from close geographic zones with low climatic variation. In general, no correlation was observed between the molecular distribution and geographic origin or climatic characteristics where the Spanish B. bassiana isolates were sampled.

Keywords: Entomopathogenic fungi LSU rDNA group I introns, Translation elongation factor gene, Thermal growth

\section{Background}

The anamorphic fungus Beauveria bassiana (Bals.) Vuill. (teleomorph: Cordyceps bassiana) is the most widely used mycopesticide for the biological control of insect pests [1,2], formulations based on this fungus being available for commercial use [3]. However, there are still many unresolved questions in our understanding of the life of fungal entomopathogens, including their population characteristics and relationships between genotypes and habitats or host-pathogen interactions [4]. For

\footnotetext{
*Correspondence: emv@usal.es

${ }^{3}$ Centro Hispano-Luso de Investigaciones Agrarias (CIALE), Departamento de Microbiología y Genética, Universidad de Salamanca, Río Duero 12, Campus de Villamayor, 37185 Salamanca, Spain

Full list of author information is available at the end of the article
}

predictable and successful application of biological control agents (BCAs) to control diseases and pests in natural environments, their biology and ecology must be well understood [5-7].

The morphological features of conidia are common tools for identification in Beauveria. Morphological and molecular studies have shown that the broad patterns of diversity in Beauveria have been accurately predicted in previous morphological studies. However, they have also shown that these approaches are insufficient to investigate species such as B. bassiana [8]. Molecular data applied to taxonomic investigations have demonstrated that B. bassiana is a species complex with several cryptic species and have corroborated their link to Cordyceps

\section{Biomed Central}


teleomorphs [8-12]. In this sense, phylogenetic studies based on nuclear ITS and elongation factor 1-alpha (EF1- $\alpha$ ) sequences have demonstrated the monophyly of Beauveria and the existence of at least two lineages within B. bassiana s.l. (sensu lato), and also that EF1- $\alpha$ sequences provide adequate information for the inference of relationships in this genus [8]. Studies on the genetic variability of BCAs such as B. bassiana are crucial for the development of molecular tools for their monitoring in the natural environment [6].

Minisatellite loci [13], random amplified polymorphism DNA (RAPD) [14], universally primed (UP) PCR [15], amplified fragment length polymorphism (AFLP) [16], isoenzyme analyses [17], or combinations of these methods [18] have provided useful polymorphisms to access genetic diversity among $B$. bassiana isolates.

Although some molecular studies have correlated $B$. bassiana genetic groups and host affiliation $[9,19]$, more recent evidence indicates that this is not the case since B. bassiana contains generalist enthomopathogens with no particular phylogenetic association with their insect host $[7,18]$, environmental factors being the prime selective forces for genotypic evolution in B. bassiana [7]. In this sense, several studies have demonstrated the association between $B$. bassiana genetic groups and Canadian [20], Brazilian [18] and world-wide [21] climatic zones.

Entomopathogenic species displayed a high degree of variability-mainly attributed to the presence of group I introns- at specific sites of the coding regions of small and large subunits of nuclear ribosomal RNA genes (SSU rDNA and LSU rDNA). Group I introns in entomopathogenic fungi were initially reported in Beauveria brongniartii LSU genes [22]. Work addressing the presence and usefulness of these non-coding elements has been reported for Beauveria. For example, Neuvéglise et al. [23] found 14 form variants of introns, differing in size and restriction patterns, at four different LSU positions from among a panel of 47 isolates of B. brongniartii, two of B. bassiana, and one of Metarhizium anisopliae from several geographic origins. Coates et al. [24] found 12 intron forms in the SSU from 35 Beauveria isolates. Wang et al. [25] analyzed the presence of group I introns in the four LSU insertion positions, designated Bb1 (also known as Ec2563), Bb2 (Ec2449), $\mathrm{Bb} 3$ (Ec2066) and Bb4 (Ec1921), and distributed a collection of $125 \mathrm{~B}$. bassiana isolates in 13 different genotypes. In that study, their sequence analyses confirmed that the introns were invariably inserted in specific target sequences, and a strong correlation between specific insertion sites and intron subgroups was also observed. In addition, the features and behaviour of these group I intron were also detected in related genera such as Cordyceps [26] and Metarhizium [27].
The present study was undertaken to investigate the genetic variability existing in a collection of 53 Spanish isolates of $B$. bassiana, obtained from different substrates or insect hosts, and 4 isolates from other European countries. The insertion patterns of group I introns at the 3'end of the LSU rDNA genes and EF1- $\alpha$ phylogenetic distribution were integrated in order to explore any possible correlation between genetic groups and geographical/climate origin, and habitat or insect host.

\section{Results}

\section{Analysis of group I introns in 3' LSU rDNA}

The 3'-end of the nuclear LSU rDNA genes of the $57 \mathrm{~B}$. bassiana isolates (Table 1) was amplified with primers I29 and M1 and four different sizes of PCR products were observed on agarose gels, ranging from 0.79 to $1.77 \mathrm{~kb}$. The sizes were as follows: about $1650 \mathrm{bp}$ for 44 isolates; 1770 bp for one isolate; 1280 bp for 9 isolates, and $790 \mathrm{bp}$ for 3 isolates. All amplicons were purified and sequenced in order to determine whether the insertion of multiple sequences, a feature described for members of this and other entomopathogenic genera, was responsible for the diversity of their lengths.

After sequencing analysis (Table 2), we observed that the smallest PCR products were detected in 3 out of the 57 isolates studied-coded Bb19, Bb50 and Bb57-indicating that these isolates had no introns, and the intronless sequence size was $790 \mathrm{bp}$; identical in composition to a homologous fragment of B. bassiana s.l. [25] described previously. The other 54 isolates exhibited introns inserted at one or more of the four possible conserved positions. Among these 54 intron-containing isolates, the insertion was as follows: 44 showed inserted sequences at positions 1 (Ec2563) and 4 (Ec1921); one isolate, $\mathrm{Bb} 51$, with a sequence size of $1770 \mathrm{bp}$, contained two introns at positions 2 (Ec2449) and 4. (Ec1921), and nine isolates contained only one intron at position 4 .

The presence/absence of introns at the 3'-end of the nuclear LSU rDNA of the 57 isolates analyzed allowed their distribution in the following genotypes: A1B2B3A4, $\mathrm{B} 1 \mathrm{~A} 2 \mathrm{~B} 3 \mathrm{~A} 4, \mathrm{~B} 1 \mathrm{~B} 2 \mathrm{~B} 3 \mathrm{~A} 4$ and $\mathrm{B} 1 \mathrm{~B} 2 \mathrm{~B} 3 \mathrm{~B} 4$ ( $\mathrm{A}=$ presence, $\mathrm{B}$ = absence; according to Wang et al. [25]). Insertion sites are numbered from 1 to 4, also following Wang's terminology [25]: Ec2563 (position 1), Ec2449 (position 2), Ec2066 (position 3) and Ec1921 (position 4). These genotypes and their distribution frequencies are shown in Table 2. Three out of the 57 isolates had no introns; nine contained one, and forty-five had two introns. Fifty-four of 57 isolates showed an inserted intron at position 4 , and 44 isolates at position 1 , whereas only one isolate had an inserted intron at position 2. None of the 57 isolates had introns at the 3 insertion site.

There was a significant correlation between belonging to an intron genotype and the mean of the optimal 
Table 1 Information concerning the Beauveria bassiana isolates analyzed in this study.

\begin{tabular}{|c|c|c|c|c|}
\hline Code & Isolate & Location & Climate & Habitat/Host (Order) \\
\hline $\mathrm{Bb} 1$ & EABb 01/145-Su & Sevilla (Spain) & M & olive \\
\hline $\mathrm{Bb} 2$ & EABb 01/160-Su & Huelva (Spain) & M & oak \\
\hline Bb3 & EABb 01/164-Su & Huelva (Spain) & M & pine \\
\hline $\mathrm{Bb} 4$ & EABb 01/168-Su & Huelva (Spain) & M & scrubland \\
\hline Bb5 & EABb 01/171-Su & Huelva (Spain) & M & cotton \\
\hline $\mathrm{Bb} 6$ & EABb 01/15-Su & Almería (Spain) & M & dessert \\
\hline $\mathrm{Bb} 7$ & EABb 01/126-Su & Cádiz (Spain) & M & olive \\
\hline $\mathrm{Bb} 8$ & EABb 01/75-Su & Almería (Spain) & M & seaside \\
\hline $\mathrm{Bb} 9$ & EABb 01/116-Su & Sevilla (Spain) & M & olive \\
\hline Bb10 & EABb 01/112-Su & Sevilla (Spain) & M & wheat \\
\hline Bb11 & EABb 01/125-Su & Cádiz (Spain) & M & fallow land \\
\hline $\mathrm{Bb} 12$ & $\mathrm{EABb} 00 / 10-\mathrm{Su}$ & Jaén (Spain) & M & olive \\
\hline $\mathrm{Bb} 13$ & $\mathrm{EABb} 00 / 11-\mathrm{Su}$ & Jaén (Spain) & M & scrubland \\
\hline Bb14 & EABb 00/13-Su & Jaén (Spain) & M & woodland \\
\hline Bb15 & $\mathrm{EABb} 00 / 16-\mathrm{Su}$ & Almería (Spain) & M & scrubland \\
\hline $\mathrm{Bb} 16$ & EABb 00/17-Su & Almería (Spain) & M & dessert \\
\hline Bb17 & EABb 01/07-Su & Córdoba (Spain) & M & meadow \\
\hline Bb18 & EABb 01/19-Su & Granada (Spain) & M & wheat \\
\hline Bb19 & EABb 01/22-Su & Córdoba (Spain) & M & scrubland \\
\hline Bb20 & EABb 01/25-Su & Córdoba (Spain) & M & olive \\
\hline Bb21 & EABb 01/27-Su & Córdoba (Spain) & M & wheat \\
\hline Bb22 & EABb 01/33-Su & Cádiz (Spain) & M & olive \\
\hline Bb23 & EABb 01/34-Su & Málaga (Spain) & M & olive \\
\hline Bb24 & EABb 01/35-Su & Málaga (Spain) & M & scrubland \\
\hline Bb25 & EABb 01/36-Su & Málaga (Spain) & M & meadow \\
\hline Bb26 & $\mathrm{EABb} 01 / 37-\mathrm{Su}$ & Málaga (Spain) & M & olive \\
\hline Bb27 & EABb 01/43-Su & Jaén (Spain) & M & olive \\
\hline Bb28 & EABb 01/45-Su & Jaén (Spain) & M & scrubland \\
\hline Bb29 & EABb 01/64-Su & Granada (Spain) & M & woodland \\
\hline Bb30 & EABb 01/73-Su & Granada (Spain) & M & scrubland \\
\hline Bb31 & $\mathrm{EABb} 01 / 76-\mathrm{Su}$ & Granada (Spain) & M & scrubland \\
\hline Bb32 & EABb 01/100-Su & Sevilla (Spain) & M & olive \\
\hline Bb33 & EABb 01/103-Su & Sevilla (Spain) & M & woodland \\
\hline Bb34 & EABb 01/105-Su & Sevilla (Spain) & M & cotton \\
\hline Bb35 & EABb 01/130-Su & Cádiz (Spain) & M & pine \\
\hline Bb36 & EABb 01/132-Su & Cádiz (Spain) & M & cotton \\
\hline Bb37 & $\mathrm{EABb} 90 / 2-\mathrm{Dm}$ & Badajoz (Spain) & M & Dociostaurus maroccanus (Orthoptera) \\
\hline Bb38 & $\mathrm{EABb} 90 / 4-\mathrm{Cb}$ & Badajoz (Spain) & M & Chortipus bicolor (Orthoptera) \\
\hline Bb39 & EABb 91/6-Ci & Badajoz (Spain) & M & Calliptamus italicus (Orthoptera) \\
\hline Bb40 & EABb 91/7-Dm & Badajoz (Spain) & M & D. maroccanus (Orthoptera) \\
\hline Bb41 & $\mathrm{EaBb} 92 / 10-\mathrm{Dm}$ & Badajoz (Spain) & M & D. maroccanus (Orthoptera) \\
\hline Bb42 & EABb 92/11Dm & Badajoz (Spain) & M & D. maroccanus (Orthoptera) \\
\hline Bb43 & EABb 93/14-Tp & Córdoba (Spain) & M & Thaumetopea pytiocampa (Lepidoptera) \\
\hline Bb44 & EABb 04/01-Tip & Sevilla (Spain) & M & Timaspis papaveris (Hymenoptera) \\
\hline Bb45 & EABb 01/88-Su & South Portugal & M & sunflower \\
\hline $\mathrm{Bb} 46$ & EABb 01/39-Su & Málaga (Spain) & M & almond \\
\hline Bb47 & EABb 01/110-Su & Sevilla (Spain) & M & holm oak \\
\hline Bb48 & EABb 04/06-Su & Córdoba (Spain) & M & cork oak \\
\hline Bb49 & EABb 04/08-Su & Córdoba (Spain) & M & hazel \\
\hline Bb50 & $\mathrm{EABb} 04 / 02-\mathrm{Su}$ & Santander (Spain) & $\mathrm{HO}$ & Ebro river \\
\hline Bb51 & EABb 04/03-Su & Santander (Spain) & $\mathrm{HO}$ & grassland \\
\hline
\end{tabular}


Table 1 Information concerning the Beauveria bassiana isolates analyzed in this study. (Continued)

\begin{tabular}{lllll}
\hline Bb52 & EABb 04/05-Su & Álava (Spain) & C & leek \\
Bb53 & EABb 04/09-Su & Madrid (Spain) & C & grassland \\
Bb54 & EABb 04/10-Su & Gerona (Spain) & M & olive \\
Bb55 & EABb 04/12-Su & Georgia & C & inculto \\
Bb56 & B. bassiana 1333 & Greece & M & Bactrocera oleae (Diptera) \\
Bb57 & B. bassiana 3395 & Poland & C & No data available \\
\hline
\end{tabular}

Code: reference as each isolate is cited in the text.

Source: reference as received from the Collection from the Department of Ciencias y Recursos Agrícolas y Forestales (CRAF) of the University of Córdoba, Spain. Climatic: zones where isolates were collected (M: subtropical Mediterranean, C: continental, HO: humid oceanic).

$\left(\mathrm{F}_{1,84}: 57.20^{\circ} \mathrm{C} ; \mathrm{P}<0.001\right)$ and highest $\left(\mathrm{F}_{1,84}: 27.39^{\circ} \mathrm{C} ; \mathrm{P}\right.$ $<0.001)$ growth temperatures, which were significantly lower in the genotype B1B2B3A4, with $\mathrm{T}_{\text {opt }}$ and $\mathrm{T}_{\max }$ values of 24.3 and $33.9^{\circ} \mathrm{C}$, respectively, than those obtained for A1B2B3A4, with $\mathrm{T}_{\text {opt }}$ of 26.7 and $\mathrm{T}_{\max }$ $35.6^{\circ} \mathrm{C}$ (data not shown).

Two different intron sequence sizes, 427 or 443 bp in length, were detected at position 4 within the 54 Beauveria isolates that bore an insertion at this site, allowing the distribution of the isolates into two sub-genotypes (Table 2). Three of these 54 isolates had a sequence of $427 \mathrm{bp}$, showing $100 \%$ identity with the 4-position intron sequence reported for B. bassiana Bb232 [25]. In 51 of the $B$. bassiana isolates, the inserted sequence length at this position was $443 \mathrm{bp}$, and four variants with few nucleotide differences were observed after alignment of these sequences, showing identity values of 98 to $100 \%$ with another sequence detected at the same position in B. bassiana Bb726 [24].

The intron sequence inserted at position 2 was only detected for Bb51, an isolate obtained in Santander (North Spain), and was 502 bp long. This intron shared 99 and $98 \%$ identity with two sequences previously detected at the same position in the LSU of B. bassiana isolates 178 and 1121 [24,25]. A 387-bp intron was identified in 44 isolates at position 1. Alignment of these sequences revealed that the 387-bp sequence was conserved in the $44 \mathrm{~B}$. bassiana isolates, where this intron was observed, and this sequence had identity values of $98 \%$ with the previously described sequence of $B$. bassiana ECBL16 [24].

The seven different $B$. bassiana intron sequences exhibited the typical characteristics of group I and no ORFs were detected. These intron sequences from $B$. bassiana were compared with other fungal intron sequences available in databases for their placement in previously reported subgroups [28]. The introns inserted at positions 2 and 4 were placed in the IC1 subgroup (one of the 15 subgroups, based on their secondary structure, described within the group I introns), and that inserted at position 1 was placed in the IE subgroup. As previously observed in group I introns [25-27], those inserted at the same site all belonged to the same subgroup. The intron sequences obtained in this work were compared with other $B$. bassiana intron sequences representing different subgroups to examine their polymorphisms (data not shown). Intron size and nucleotide identity differences were observed but $\mathrm{P}, \mathrm{Q}$,

Table 2 Genotypes derived from the presence/absence of introns in LSU rDNA genes for 57 Beauveria bassiana isolates and types of intron sequences.

\begin{tabular}{|c|c|c|c|c|c|}
\hline \multirow[b]{2}{*}{$\begin{array}{l}\text { Genotype * } \\
\text { (\%) }\end{array}$} & \multirow[b]{2}{*}{ Isolate code } & \multirow[b]{2}{*}{ No. isolates } & \multicolumn{3}{|c|}{ GenBank } \\
\hline & & & $\begin{array}{l}\text { position } 1 \\
\text { (Ec2563) }\end{array}$ & $\begin{array}{l}\text { position } 2 \\
\text { (Ec2449) }\end{array}$ & $\begin{array}{l}\text { position } 4^{* *} \\
\text { (Ec1921) }\end{array}$ \\
\hline \multirow[t]{4}{*}{ A1B2B3A4 } & Bb2-5, Bb32-33, Bb35, Bb45, Bb48-49 & 10 & EF115312 & & EF115308 (433a) \\
\hline & Bb1, Bb6-12, Bb14-17, Bb20-21, Bb23-31, & & & & \\
\hline & Bb34, Bb36, Bb41-42, Bb44, Bb46-47, & & & & \\
\hline & Bb52-54, Bb56 & 34 & EF115312 & & EF115307 (443b) \\
\hline B1A2B3A4 & Bb51 & 1 & & EF115313 & EF115309 (427) \\
\hline \multirow[t]{3}{*}{ B1B2B3A4 } & $\mathrm{Bb} 13, \mathrm{Bb} 18$ & 2 & & & EF115310 (443c) \\
\hline & Bb22, Bb37, Bb39-40, Bb43 & 5 & & & EF115311 (443d) \\
\hline & Bb38, Bb55 & 2 & & & EF115309 (427) \\
\hline B1B2B3B4 & $\mathrm{Bb} 19, \mathrm{Bb} 50, \mathrm{Bb} 57$ & 3 & - & - & - \\
\hline
\end{tabular}

${ }^{*} \mathrm{~A}$, presence, and B, absence of a given intron at the 3'-end of the nuclear LSU rDNA genes. Numbers 1-4 represent insertion sites Ec2563, Ec2449, Ec2066 and Ec1921, respectively, as previously described [25].

** Sequence types inserted at position 4 (Ec1921) are indicated by their sizes in bp, followed by a letter for identical sizes (i.e., $443 a$, b, c, d) to indicate small differences in their composition. 
$\mathrm{R}$ and $\mathrm{S}$ motif elements, which are needed for the formation of the secondary structure of group I introns [29], were highly conserved among the introns inserted at the same site, particularly for position 1 . The highest polymorphism was observed in introns inserted at 2, the P1-P3 helices being the source of this variation, and at 4, in the P5, P6 and P8 helices.

The MP tree obtained after an alignment of the 7 different intron sequence types identified from 57 B. bassiana isolates and another 24 GenBank-deposited sequences, which represent intron sequences from $M$. anisopliae, B. bassiana and Cordyceps profilica, together with the subsequent phylogenetic analysis are shown in Figure 1. The tree reveals the separation of four independent groups, supported by high bootstrap values, corresponding to the four positions reported previously [25]: Ec1921 (position 4), Ec2066 (position 3), Ec2449 (position 2) and Ec2563 (position 1), where intron insertions occurred. The tree shows that the sequence group located at position 4 is closer to those at position 2 and both contain IC1 subgroup introns. Similarly, position 3 sequences are closer to position 1 sequences, and both groups have IE subgroup introns. Within position 4, Cordyceps and Metarhizium were separated from Beauveria sequences and formed an independent group, supported by a bootstrap value of $100 \%$. In addition, the five different Beauveria sequences obtained here were separated into two of the four observed groups at this position, supported by bootstrap values of $94 \%$ and $60 \%$. This separation was in accordance with the two sequence sizes detected: 443 and 427-bp in length. However, the four different sequence types detected for 443-bp-sized introns were not separated after phylogenetic analysis.

\section{EF1- $\alpha$ gene analysis}

With the exception of isolate $\mathrm{Bb} 49$, where no amplification was observed, all isolates afforded PCR products of $1.1 \mathrm{~kb}$ for the EF1- $\alpha$ gene with the primers tef1 fw and 1750-R. Eleven different EF1- $\alpha$ gene sequences were identified among the 56 isolates. The alignment and comparison of these 11 sequences and another 18 GenBank-deposited sequences, representing different lineages from B. bassiana s.s. (sensu stricto), B. brongniartii and $B$. bassiana clade $C[7,8,12]$, produced 1757 aligned positions, with 1542 constant characters and 114 parsimony-informative characters. The MP tree is shown in Figure 2. Of the 56 isolates analyzed, 94.6\% (53 isolates) were located in the B. bassiana s.s. clade, and $5.4 \%$ ( 3 isolates) in clade C, which includes $B . c f$. (uncertain taxonomy) bassiana isolates. Within B. bassiana s.s., the 53 isolates analyzed in this study were separated in five subgroups (Eu-7, Eu-8 and Eu-9 with isolates from Spain and Portugal; Eu-3 from Spain,
France and Denmark; and Wd-2 with world-wide distribution), supported by bootstrap values higher than $50 \%$.

\section{Integration of intron insertion patterns and EF1- $\alpha$ phylogenetic distribution}

In order to assess the phylogenic distribution of the different intron configuration types, they were mapped on the EF1- $\alpha$ tree (Figure 2). All 53 B. bassiana s.s. isolates showed an intron IC1 inserted at position 4. However, the IE intron inserted at position 1 was only present in the 10 isolates from subgroup $\mathrm{Eu}-7$ and 33 out of 39 isolates from subgroup $\mathrm{Wd}-2$. In particular, this subgroup included most of the Spanish isolates of B. bassiana forming an EF1- $\alpha$ phylogenetic group with isolates 681 from Romania and 792 from the USA [8] but displaying two different intron insertion models. Bb51 showed a unique intron insertion pattern, with an IC1 intron at position 2, and located separately in the Eu-9 subgroup. No introns were detected at any position in the three B. cf. bassiana isolates from clade C.

No correlation between EF1- $\alpha$ phylogenetic groups and insect host was observed. Although Eu-7 subgroup did not included isolates of insect origin, the Wd-2 subgroup grouped isolates collected from Diptera, Hymenoptera, Lepidoptera and Orthoptera. Moreover, Wd-2 isolates from Orthoptera displayed different intron insertion models (i.e., Bb37, Bb39 and Bb40, and Bb42).

Forty-nine Spanish and one Portuguese isolates of $B$. bassiana s.s. were collected from subtropical Mediterranean climate zones and were distributed in the $\mathrm{Eu}-7$, Eu-3, Wd-2 and Eu-8 subgroups. Two Spanish isolates, Bb52 and Bb53, were collected from continental climate locations and were placed within subgroups $\mathrm{Eu}-7$ and Wd-2, respectively. The only B. bassiana s.s. isolate from a humid oceanic climate included in this work, Bb51 from Santander, displayed a characteristic intron insertion model and formed the EF1- $\alpha$ subgroup Eu-9. In addition, Bb51 produced smaller conidia than the rest of $B$. bassiana isolates, this morphological feature being statistically significant (data not shown). Nevertheless, other isolate from the same climatic zone, Bb50, was grouped with other European isolates in B. cf. bassiana clade $\mathrm{C}$.

\section{Discussion}

In the present study, we have identified different $B$. bassiana genotypes and phylogenetic subgroups in a collection of 57 isolates of this fungus, based on intron insertion patterns and EF1- $\alpha$ phylogenies, respectively.

The variability in group I introns from rDNA genes has been used as a molecular tool for the identification of polymorphisms in entomopathogenic fungi [23,30,31]. Our study of B. bassiana LSU rDNA identified 99 introns among the 57 isolates analyzed. Four specific 


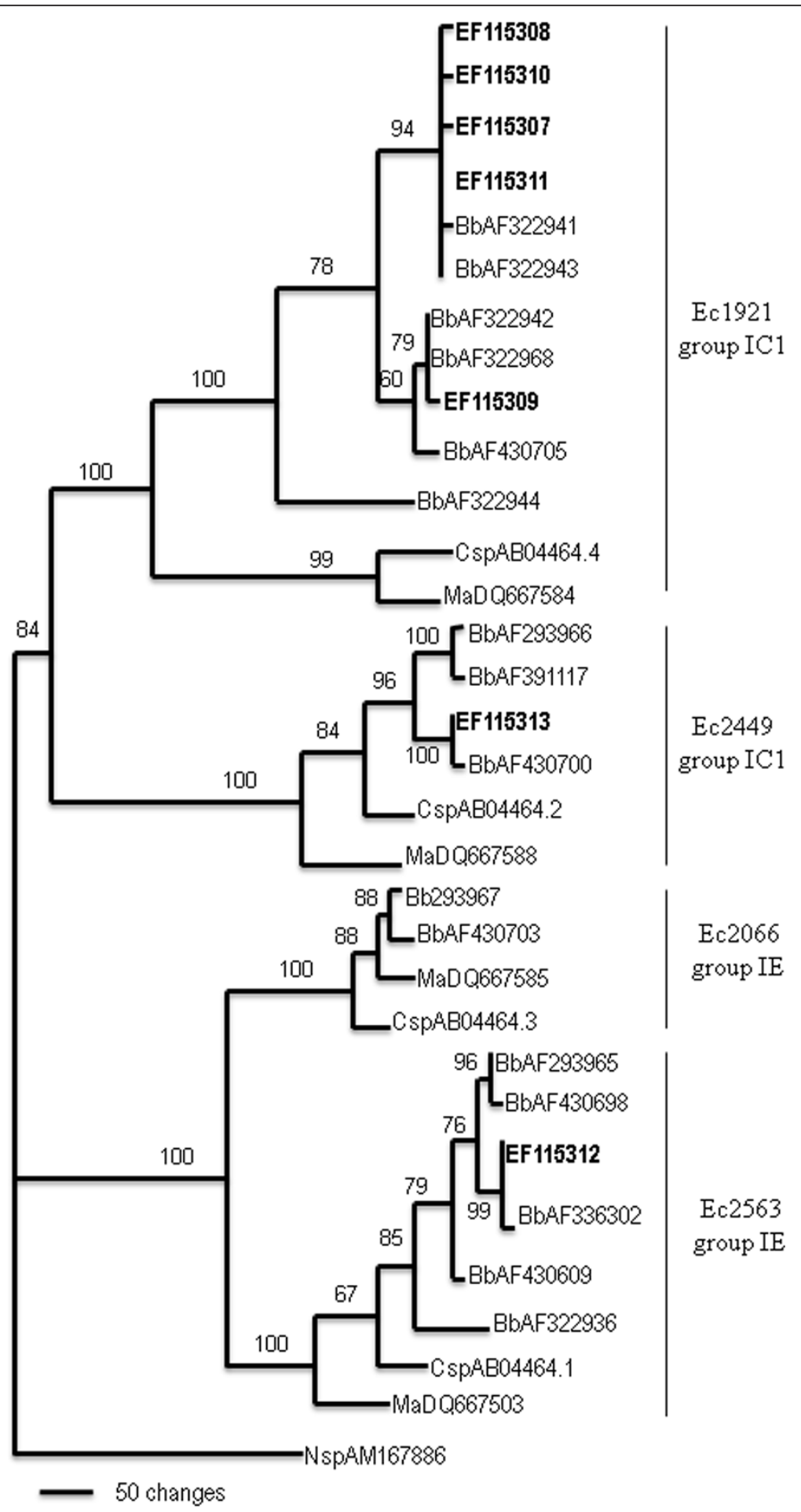

Figure 1 Phylogenetic analysis of group I introns inserted in the LSU rDNA genes of entomopathogenic fungi. The MP tree was generated by parsimony analysis after heuristic searches (TBR option). A bootstrap full heuristic analysis, with bootstrap intervals from 1000 replications and nodes supported in $>50 \%$ of bootstrap replicates, was generated using the PAUP 4.0 program. Branch lengths are proportional to the number of changes. Seven different intron sequence types (bolded) identified from 57 B. bassiana isolates were aligned with 24 representative intron sequences from Metarhizium anisopliae (Ma), Beauveria bassiana (Bb) and Cordyceps profilica (Csp), and an intron sequence from Naegleria sp. (Nsp) was used as outgroup. The four group I intron insertion positions are shown as Ec1921 (position 4), Ec2066 (position 3), Ec2449 (position 2) and Ec2563 (position 1). 


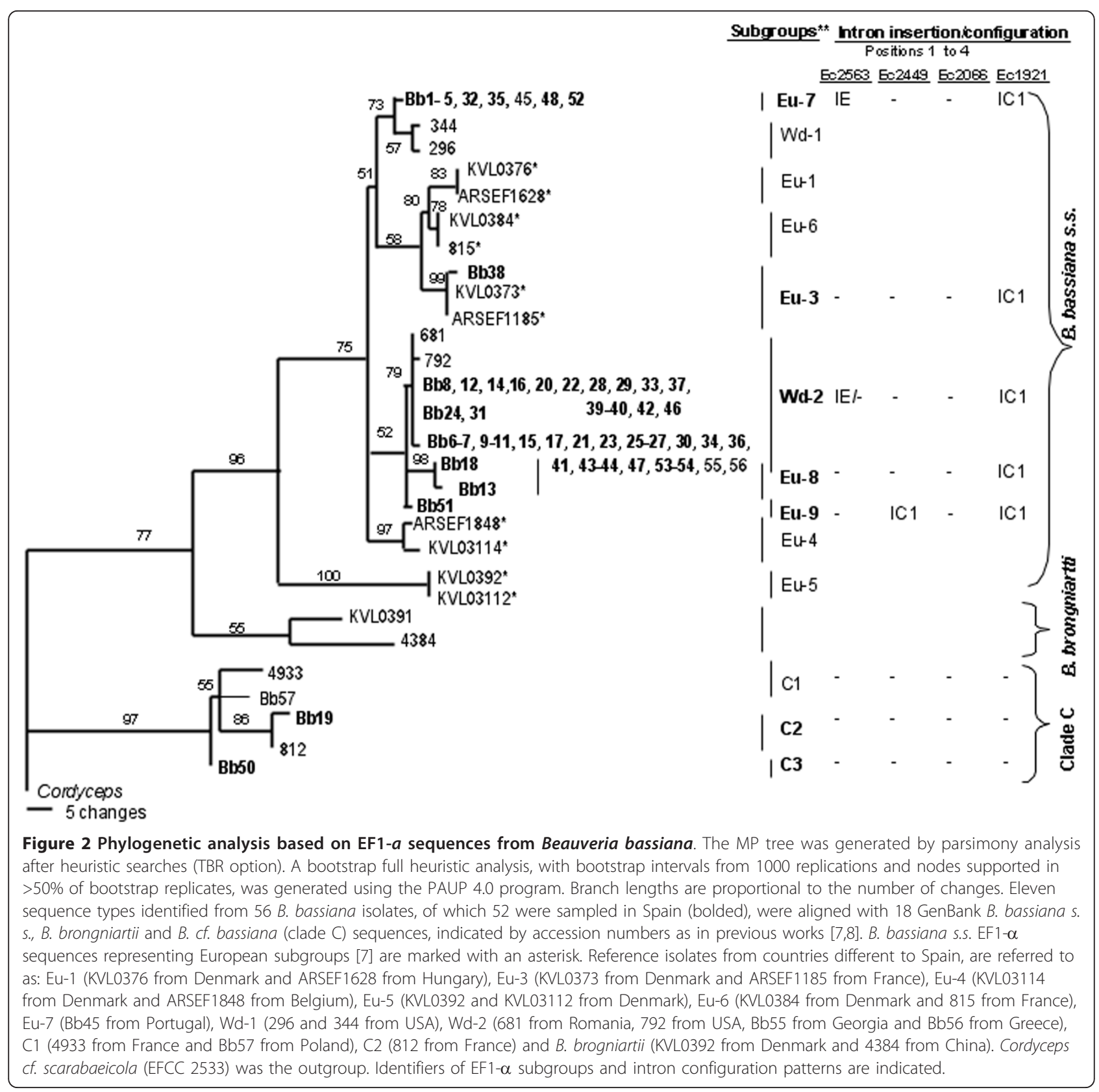

sites of intron insertion have been described previously in Beauveria species [23,25], but in our collection introns were only detected at positions 1,2 or 4 .

Particularly, our study shows that $100 \%$ of B. bassiana s.s. isolates had an intron inserted at position 4 . This position was also preferential for intron insertion (84.4\%) in a population of 125 B. bassiana isolates [25]. The number of introns found in the 57 isolates was in agreement with the 199 introns detected in $125 \mathrm{~B}$. bassiana isolates by Wang et al. [25]; the 44 introns detected in $26 \mathrm{M}$. anisopliae isolates by Márquez et al. [31], and the 69 introns found in 28 representative members of the genus Cordyceps by Nikoh and Fukatsu [26]. However, only four intron insertion patterns were present in our $B$. bassiana collection while greater variability was found in other studies: 13, 7 and 9 insertion patterns within 125 B. bassiana [25], $26 \mathrm{M}$. anisopliae [31] and 47 B. brongniartii [23] isolates, respectively.

The MP tree based on intron sequences shows that they were distributed in four large groups, with bootstrap values of $100 \%$, corresponding to four insertion positions (Figure 1). As could be expected [25,28], the introns inserted at the same site always belonged to the same subgroup: IC1 at positions 2 and 4, and IE at position 1 . 
Although the origin and transmission mechanisms of group I introns have generated controversy [26], this distribution of sequences is in agreement with previously reported observations [25] and means that introns inserted at the same position have a monophyletic origin and are transmitted vertically. In subsequent events intron speciation and diversification take place as occurs at position 4, where B. bassiana introns are separated from Metarhizium and Cordyceps introns, and two $B$. bassiana IC1 sequence sizes were located in two different sub-clades, supported by high bootstrap values.

Rehner and Buckley's study [8] based on EF1- $\alpha$ and ITS phylogenies has revealed that i) six clades can be resolved within Beauveria (A-F) and, excepting those corresponding to B. bassiana (A and C), they are closely to species previously described on the basis of their morphology, and ii) B. bassiana s.s. (A) was determined almost entirely from nucleotide variation at EF1- $\alpha$. Further phylogenetic studies carried out with nuclear and/or mitochondrial DNA regions of B. bassiana from all continents have served to resolve lineage diversity within this species $[7,12,18,21]$. Since phylogenetic species by continent and in the order of their discovery have been designated previously [7], we followed this nomenclature to refer the new phylogenetic subgroups identified among the Spanish B. bassiana s.s. isolates as $\mathrm{Eu}-7, \mathrm{Eu}-8$ and Eu-9. The results obtained from MP analyses (Figure 2), using a $1.1 \mathrm{~kb}$ fragment of the EF1$\alpha$ gene from 56 isolates from our collection, confirmed that 53 isolates were B. bassiana s.s. (A), and three isolates grouped in three different phylogenetic subgroups within B. cf. bassiana (C). As in a previous study [7], the collection of Spanish isolates of B. bassiana s.s. was separated in five phylogenetic subgroups. However, only isolate Bb38, sampled from insects, was grouped with one $(\mathrm{Eu}-3)$ of the five phylogenetic species proposed by those authors working with a Danish collection of $B$. bassiana s.s. [7], including insect isolates only. Interestingly, three phylogenetic subgroups (Eu-7, Eu-8 and Eu9) were only formed by isolates from Spanish and Portuguese isolates. However, most of the isolates in our collection (39 out of 56) were grouped with isolates from Romania and the USA in the world-wide phylogenetic subgroup Wd-2, which includes isolates from Europe, Africa and North America [8].

When the different intron insertion patterns were mapped on the B. bassiana EF1- $\alpha$ phylogeny (Figure 2), the existence of a same intron genotype in a given phylogenetic subgroup could be indicative of its clonal origin as it is the case of Eu-7 and Eu-8. Previous studies have shown that Eu-3, where Bb38 is located, is a clonal group [7]. Isolate Bb51 was the only member of Eu-9 and the separated phylogenetic grouping of this isolate is supported by a characteristic intron insertion pattern and the production of statistically significant smaller conidia than those from any other intron genotype (data not shown). The two different intron genotypes observed among the isolates from the complex phylogenetic subgroup Wd-2, may indicate that homologous recombination is involved in the IE intron loss at position 1. Previous studies have shown frequent intron losses of group I introns in the nuclear rDNAs of Cordyceps [26]. Recently, a low frequency of sexual reproduction was observed in Eu-1 [7]; this could also be the case of Wd-2 where the absence of an IE intron at position 1 was only observed in 6 out of 39 isolates of this phylogenetic subgroup.

The genetic diversity of Spanish B. bassiana s.s. isolates was compared in relation to their hosts and geographical provenance and according to the latter view [21], no general correlation can be observed between the molecular variability among isolates and host and/or geographical origin. Although most of the isolates in our study were collected from soil, 8 out of 9 isolates from insects were grouped together in the subgroup Wd-2 although they derived from different insect orders. Phylogenetic subgroups only indicated a tenuous dependence upon geographic origin (i.e., Bb2-5 located in $\mathrm{Eu}-$ 7 or Bb23-26 and Bb29-31 located in Wd-2). A recent phylogeographic report [18] has provided evidence that the genetic distance of Brazilian B. bassiana isolates correlates with geographical distance, suggesting that according to Rehner's study [12] allopatry plays an important role in the phylogenetic diversification of $B$. bassiana. The authors of another recent study [7] concluded that multiple phylogenetic species of B. bassiana s.s. co-exist in sympatry within the limited natural habitat of a bordering hedgerow. We observed that isolates sampled in close locations were placed in different phylogenetic subgroups (i.e., Bb35 and Bb36, from Cádiz, belong to $\mathrm{Eu}-7$ and $\mathrm{Wd}-2$, respectively; and $\mathrm{Bb} 38$, and $\mathrm{Bb} 39-40$ and $\mathrm{Bb} 42$, from Badajoz, group within $\mathrm{Eu}-3$ and $\mathrm{Wd}-2$, respectively, $\mathrm{Bb} 39-40$ and $\mathrm{Bb} 42$ having different intron genotypes). According to Meyling's study [7], the high phylogenetic diversity of the Spanish isolates of B. bassiana s.s. could be explained by the untilled habitats where most of them were sampled (i.e., olive, oak, pine, meadow or scrubland).

Previous studies have suggested that the saprophytic phase of entomopathogenic fungi exerts evolutionary pressure on the genotype and that adaptation to a habitat type is associated with their environmental preferences [20]. Recent studies have also pointed out the importance of climatic conditions in the prevalence and distribution of B. bassiana genotypes [21]. Our study was carried out on 51 isolates from subtropical Mediterranean climate locations that were distributed within the phylogenetic subgroups $\mathrm{Eu}-3, \mathrm{Eu}-7, \mathrm{Eu}-8, \mathrm{Wd}-2$ and 
clade C; 4 isolates were from continental climate sites and grouped in Eu-7, Wd-2 and clade C; and 2 isolates came from a humid oceanic climate zone, being located in Eu-9 and clade $\mathrm{C}$. Interestingly, the only B. bassiana s.s. from a humid oceanic climate was the singular isolate $\mathrm{Bb} 51$. The fact that isolates from Mediterranean or continental climates overlapped in different phylogenetic subgroups, could be due to lower differences among the abiotic conditions existing in Spain, a country covering far smaller geographical surface and with much less variability than that considered in other Canadian, Brazilian or world-wide studies where phylogenetic species showed a better correlation with climate characteristics [21], biogeographic distribution [18] and habitat [20]. In a thermal growth study [20] it was described that $B$. bassiana genetic groups from three different habitats in Canada were associated with temperature preferences. When we explored the thermal preferences within a set of Spanish B. bassiana s.s. isolates belonging to the two main intron genotypes (A1B2B3A4 and B1B2B3A4) and four phylogenetic EF1- $\alpha$ subgroups (data not shown), a correlation between intron genotypes and the mean optimal and maximum temperatures for growth was observed, both growth temperatures being significantly lower in the B1B2B3A4 genotype with respect to A1B2B3A4. However, no correlation was observed between thermal preferences and the climatic origin of the Spanish B. bassiana isolates.

\section{Conclusion}

Four intron genotypes, and five and three phylogenetic subgroups within B. bassiana s.s. and B. cf. bassiana (clade $C$ ) have been identified, respectively, in a collection of 57 B. bassiana isolates -53 from Spain. The highest polymorphism was observed in introns inserted at positions 2 and 4. All B. bassiana s.s. displayed an IC1 intron inserted at position 4. Integration of intron insertion patterns and EF1- $\alpha$ phylogenetic distribution served to demonstrate the monophyletic origin and vertical transmission of introns inserted at the same site. In subsequent events intron speciation and diversification take place as occurs at site 4 , where $B$. bassiana introns are separated from Metarhizium and Cordyceps introns. No general correlation was observed between the molecular data and insect host, but a tenuous correlation was detected with the geographic origins. The high phylogenetic diversity of the Spanish isolates of B. bassiana s.s. could be due to the untilled habitats where most of them were sampled.

\section{Methods}

\section{Fungal isolates and morphological studies}

The 57 isolates of B. bassiana used in this study were selected from a Spanish collection of 960 records at the
CRAF (Ciencias y Recursos Agrícolas y Forestales) Department of the University of Cordoba (Córdoba, Spain), representing different geographic origins, habitats/hosts and climates. Fifty-three Spanish isolates were studied, 51 of them being collected from subtropical Mediterranean climate zones -characterized by warm to hot, dry summers and mild to cool, wet winters- and 2 from a humid oceanic climate. Forty-five out of these 53 isolates were from soil, most of them from poorly tilled or untilled fields (i.e., olive, oak, pine or scrubland) and 8 were isolated from insects. Information about these isolates is provided in Table 1. All fungal isolates were derived from single conidial spores grown on Malt Extract Agar plates (MEA, Difco Becton Dickinson, Sparks, MD).

\section{DNA extraction, PCR amplification, and sequencing}

Mycelia for DNA extraction were obtained as previously described [31]. Total DNA was extracted using the method previously described [32].

Two nuclear gene regions, LSU rDNA and EF1- $\alpha$, were amplified, sequenced and analyzed. The 3'-end of the nuclear LSU rDNA cluster was also amplified with primers I29 (5'-CTGCCCAGTGCTCTGAATGTC-3') [25] and M1 (5'-GGTAAAACTAACCTGTCTCACG-3') [31] for the 57 isolates of Beauveria included in the study. The distribution of putative introns was investigated using the following combinations of previously described primers: I29-I38, I31-I32, I21-I22 and E23-M1 [25,31]. A 1100 bp fragment spanning the 3' $2 / 3$ of the EF1- $\alpha$ gene was amplified with primers tef1 fw (5'GTGAGCGTGGTATCACCA-3') [33] and 1750-R (5'GACGCATGTCACGGACGGC-3') for all isolates, except $\mathrm{Bb} 49$. The oligonucleotide 1750- $\mathrm{R}$ was designed at the 3'-end of an alignment of Beauveria EF1- $\alpha$ genes obtained from databases. PCR was performed in a total volume of $50 \mu \mathrm{l}$ containing $25 \mathrm{ng}$ of genomic DNA and $0.20 \mu \mathrm{M}$ concentrations of each of the above primers, using the Taq polymerase system (Biotools B\&M Labs, Madrid, Spain) and following the manufacturer's instructions. The amplification program included an initial denaturing cycle of $1 \mathrm{~min}$ at $94^{\circ} \mathrm{C}$, followed by 35 cycles of $1 \mathrm{~min} 30 \mathrm{~s}$ at $94^{\circ} \mathrm{C}, 2 \mathrm{~min}$ (for EF1- $a$ ) or $2 \mathrm{~min}$ $30 \mathrm{sec}$ for (LSU rDNA) at 55 (for EF1-a) or $57^{\circ} \mathrm{C}$ (for LSU rDNA) and $3 \mathrm{~min}$ at $72^{\circ} \mathrm{C}$, and a final extension step of $7 \mathrm{~min}$ at $72^{\circ} \mathrm{C}$ in a PCR System 9700 Genetic Thermal Cycler (Applied Biosystems, Foster City, CA). The PCR products were electrophoresed on 1\% agarose gels buffered with $1 \times \mathrm{TAE}$ [34] and stained with ethidium bromide. A 100-bp ladder molecular weight standard (Roche Mannheim, Mannheim, Germany) was also used.

The PCR products were purified from agarose gels using the Geneclean II $\mathrm{kit}^{\circledR}$ system (Q-Biogene, 
Carlsbad, CA), following the manufacturer's protocol. DNA sequences were obtained using an automated ABI 377 Prism Sequencer (Applied Biosystems, Foster City, CA) with fluorescent terminators at the Department of Microbiology and Genetics of the University of Salamanca. All PCR products were sequenced in both directions, using amplification primers and internal primers when necessary.

The intron and EF1- $\alpha$ sequences obtained in this study were deposited in the GenBank database. Intron and EF1- $\alpha$ sequence accession numbers are available in Table 2 and additional file 1 respectively.

\section{Molecular analyses}

The presence or absence of introns at the 3'-end of the nuclear LSU rDNA of each isolate was determined by detecting previously described target sequences [25]. In order to compare the results obtained in this study with the $B$. bassiana genotypes based on previously reported intron insertion patterns in the LSU rDNA gene, Wang's terminology was used [25]. The intron sequences detected in each insertion point were aligned with representative Beauveria sequences to examine their polymorphisms and to identify conserved motifs. Intron subgroups were determined by comparison with representative secondary structures from previous studies $[25-27,30]$.

Intron and EF1- $\alpha$ sequences were analyzed separately. Published sequences for isolates included within the genera Beauveria, Metarhizium and Cordyceps were retrieved from GenBank and included in the alignments. Alignments were generated using the MegAlign (DNASTAR package, 1989-92, London, UK) and the CLUSTALX 1.81 program [35]. Phylogenetic analyses were carried out with the PAUP* version 4.0 b10 program. Gaps, encoded as missing data, and uninformative characters were excluded from the analyses. Most-parsimonious (MP) trees were obtained for intron and EF1- $\alpha$ data from heuristic searches using TBR branch-swapping [36], and all MP trees were summarized in a single tree in which all branch lengths equal to zero were collapsed by polytomies. An intron sequence of Naegleria sp. (AM167886) and the EF1- $\alpha$ gene of Cordyceps cf. scarabaeicola (AY531967) were used as outgroups in the analysis of intron and EF1$\alpha$ sequences, respectively. A bootstrap full heuristic analysis consisting of 1000 replicates was performed, and a 50\% majority rule tree was produced.

\section{Additional material}

Additional file 1: Table of GenBank accession numbers of EF1- $a$ sequences obtained in this study from 57 Beauveria bassiana isolates and $\mathrm{EF} 1-\alpha$ subgroups.

\section{Acknowledgements}

This manuscript is in memoriam of Marcela Márquez, deceased in the course of this research. This work has been funded by the Spanish Ministry of Education and Science, projects AGL2004-06322-C02-02/AGR and AGL20080512/AGR; and Junta de Castilla y León, project GR67.

\section{Author details}

'Departamento de Ciencias y Recursos Agrícolas y Forestales, Universidad de Córdoba, Edificio C4 Celestino Mutis, Campus Rabanales, 14071 Córdoba, Spain. ${ }^{2}$ Area de Genética. Departamento de Microbiología y Genética, Universidad de Salamanca, Edificio Departamental lab 324, Plaza Doctores de la Reina s/n, 37007 Salamanca, Spain. ${ }^{3}$ Centro Hispano-Luso de Investigaciones Agrarias (CIALE), Departamento de Microbiología y Genética, Universidad de Salamanca, Río Duero 12, Campus de Villamayor, 37185 Salamanca, Spain.

\section{Authors' contributions}

IGJ carried out the laboratory work related to EF1-a. MM and EAI carried out the laboratory work on introns. EQM and CSA provided the B. bassiana isolates. In addition, EQM and AOU participated in genomic DNA extraction. EM conceived the design of the study and helped to write the manuscript. $\mathrm{RH}$ participated in the design and coordination of the study, in the sequence analyses and wrote the manuscript. All authors have read and approved the final manuscript.

Received: 26 October 2010 Accepted: 26 April 2011

Published: 26 April 2011

\section{References}

1. Wraight SP, Carruthers Rl, Jaronski ST, Bradley CA, Garza CJ, GalainiWraight S: Evaluation of the entomopathogenic fungi Beauveria bassiana and Paecilomyces fumosoroseus for microbial control of the silverleaf whitefly, Bemisia argentifolii. Biol Cont 2000, 17:203-217.

2. Quesada-Moraga E, Maranhao EAA, Valverde-García P, Santiago-Álvarez C: Selection of Beauveria bassiana isolates for control of the whiteflies Bemisia tabaci and Trialeurodes vaporariorum on the basis of their virulence, thermal requirements and toxicogenic activity. Biol Cont 2006 36:274-287.

3. Wraight SP, Jackson MA, de Kock SL: Production, stabilization and formulation of fungal biocontrol agents. In Fungi as Biocontrol Agents Progress, Problems and Potential. Edited by: Butt TM, Jackson C, Magan N. Wallingford, UK: CAB International; 2001:253-287.

4. Enkerli J, Widmer F: Molecular ecology of fungal entomopathogens: molecular genetic tools and their applications in population and fate studies. Biocontrol 2010, 55:17-37.

5. Meyling NV, Eilenberg J: Ecology of the entomopathogenic fungi Beauveria bassiana and Metarhizium anisopliae in temperate agrosystems: potential for conservation biological control. Biol Cont 2007, 43:145-155.

6. Kouvelis VN, Ghikas DV, Edgington S, Typas MA, Moore D: Molecular characterization of isolates of Beauveria bassiana obtained from overwintering and summer populations of Sunn Pest (Eurygaster integriceps). Lett Appl Microbiol 2008, 46:414-420.

7. Meyling NV, Lübeck M, Buckley EP, Eilenberg J, Rehner SA: Community composition, host range and genetic structure of the fungal entomopathogen Beauveria in adjoining agricultural and seminatural habitats. Mol Ecol 2009, 18:1282-1293.

8. Rehner SA, Buckley E: A Beauveria phylogeny inferred from nuclear ITS and EF1-a sequences: evidence for cryptic diversification and links to Cordyceps teleomorphs. Mycologia 2005, 97:84-98.

9. Gaitan A, Valderrama AM, Saldarriaga G, Vélez P, Bustillo A: Genetic variability of Beauveria bassiana associated with the coffee berry borer Hypothenemus hampei and other insects. Mycol Res 2002, 106:1307-1314.

10. Aquino de Muro M, Elliott S, Moore D, Parker BL, Reid W, Bouhssini M: Molecular characterisation of Beauveria bassiana isolates obtained from overwintering sites of sunn pests (Eurygaste and Aelia species). Mycol Res 2005, 109:294-306

11. Devi KU, Reineke A, Reddy NNR, Rao CUM, Padmavathi J: Genetic diversity, reproductive biology, and speciation in the entomopathogenic fungus Beauveria bassiana (Balsamo) Vuillemin. Genome 2006, 49:495-504. 
12. Rehner SA, Posada F, Buckley EP, Infante F, Castillo A, Vega FE: Phylogenetic origins of African and Neotropical Beauveria bassiana s.l.. pathogens of the coffee berry borer, Hypothenemus hampei. J Invertebr Pathol 2006, 93:11-21.

13. Coates $B S$, Hellmich RL, Lewis $L C$ : A minisatellite from the filamentous ascomycete Beauveria bassiana shows allelic variability independent of host range and geographic origin. Genome 2002, 45:125-132.

14. Castrillo LA, Vanderberg JD, Wraight SP: Strain-specific detection of introduced Beauveria bassiana in agricultural fields by use of sequencecharacterized amplified region markers. J Invertebr Pathol 2003, 82:75-83.

15. Meyling NV, Eilenberg J: Occurrence and distribution of soil borne entomopathogenic fungi within a single organic agroecosystem. Agric Ecosyst Environ 2006, 113:336-341.

16. Aquino de Muro M, Mehta S, Moore D: The use of amplified fragment length polymorphism for molecular analysis of Beauveria bassiana isolates from Kenya and other countries, and their correlation with host and geographical origin. FEMS Microbiol Lett 2003, 229:249-257.

17. St Leger RJ, Allee LL, May R, Staples RC, Roberts DW: World-wide distribution of genetic variation among isolates Beauveria spp. Mycol Res 1992, 96:1007-1015.

18. Fernandes EKK, Moraes AML, Pacheco RS, Rangel DEN, Miller MP, Bittencourt VREP, Roberts DW: Genetic diversity among Bazilian isolates of Beauveria bassiana: comparisons with non-Brazilian isolates and other Beauveria species. J Appl Microbiol 2009, 107:760-774.

19. Berreta MF, Lecuona RE, Zandomeni RO, Grau O: Genotyping isolates of the entomopathogenic fungus Beauveria bassiana by RAPD with fluorescent labels. J Inverteb Pathol 1998, 71:145-150.

20. Bidochka MJ, Menzies FV, Kamp AM: Genetic groups of the insectpathogenic fungus Beauveria bassiana are associated with habitat and thermal growth preferences. Arch Microbiol 2002, 178:531-537.

21. Ghikas DV, Kouvelis VN, Typas MA: Phylogenetic and biogeographic implications inferred by mitochondrial intergenic region analyses and ITS1-5.8S-ITS2 of the entomopathogenic fungi Beauveria bassiana and B. brongniartii. BMC Microbiol 2010, 10:174

22. Neuvéglise C, Brygoo Y: Identification of group-I introns in the $28 \mathrm{~S}$ rDNA of the entomopathogenic fungus Beauveria brongniartii. Curr Genet 1994, 27:38-45.

23. Neuvéglise C, Brygoo Y, Riba G: 28 S rDNA group I introns: a powerful tool for identifying strains of Beauveria brongniartii. Mol Ecol 1997, 6:373-381.

24. Coates BS, Hellmich RL, Lewis LC: Nuclear small subunit rRNA group I intron variation among Beauveria spp. provide tools for strain identification and evidence of horizontal transfer. Curr Genet 2002, 41:414-424.

25. Wang CS, Li Z, Typas MA, Butt TM: Nuclear large subunit rDNA group I intron distribution in a population of Beauveria bassiana strains: phylogenetic implications. Mycol Res 2003, 107:1189-1200.

26. Nikoh N, Fukatsu T: Evolutionary dynamics of multiple group I introns in nuclear ribosomal RNA genes of endoparasitic fungi of the genus Cordyceps. Mol Biol Evol 2001, 18:1631-1642.

27. Pantou M, Mavridou A, Typas MA: IGS sequence variation, group-I introns and the complete nuclear ribosomal DNA of the entomopathogenic fungus Metarhizium: excellent tools for isolate detection and phylogenetic analysis. Fungal Genet Biol 2003, 38:159-174.

28. Michel F, Westhof E: Modelling of the three-dimensional architecture of group I catalytic introns based on comparative sequence analysis. J Mol Biol 1990, 216:585-610.

29. Cech RT: Conserved sequences and structures of group I introns: building an active site for RNA catalysis. Gene 1988, 73:259-271.

30. Mavridou A, Cannone J, Typas MA: Identification of group I introns at three different positions within the 28S rDNA gene of the entomopathogenic fungus Metarhizium anisopliae var.anisopliae. Fungal Genet Biol 2000, 31:79-90.

31. Márquez M, Iturriaga EA, Quesada-Moraga E, Santiago-Álvarez C, Monte E, Hermosa R: Detection of potentially valuable polymorphisms in four group I intron insertion sites at the $3^{\prime}$-end of the LSU rDNA genes in biocontrol isolates of Metarhizium anisopliae. BMC Microbiol 2006, 6:77.

32. Möller EM, Bahnweg G, Sandermann H, Geiger HH: A simple and efficient protocol for the isolation of high molecular weight DNA from filamentous fungi, fruit bodies, and infected plant tissues. Nucleic Acids Res 1992, 20:6115-6116.
33. O'Donnell K, Cigelnik E, Nirenberg HI: Molecular systematics and phylogeography of the Gibberella fujikuroi species complex. Mycologia 1998, 90:465-493.

34. Sambrook J, Fritsch EF, Maniatis T: Molecular Cloning: A Laboratory Manual. New York: Cold Spring Harbor Laboratory Press; 1989.

35. Thompson JD, Gibson TJ, Plewniak F, Jeanmougin F, Higgins DG: The Clustal $\times$ windows interface: flexible strategies for multiple sequence alignment aided by quality analysis tools. Nucleic Acids Res 1997, 24:4876-4882.

36. Felsenstein J: Confidence limits on the bootstrap: an approach using the bootstrap. Evolution 1985, 39:783-791.

doi:10.1186/1471-2180-11-84

Cite this article as: Garrido-Jurado et al:: Genetic analyses place most Spanish isolates of Beauveria bassiana in a molecular group with wordwide distribution. BMC Microbiology 2011 11:84.

\section{Submit your next manuscript to BioMed Central and take full advantage of:}

- Convenient online submission

- Thorough peer review

- No space constraints or color figure charges

- Immediate publication on acceptance

- Inclusion in PubMed, CAS, Scopus and Google Scholar

- Research which is freely available for redistribution

Submit your manuscript at www.biomedcentral.com/submit
C) Biomed Central 